[Chem. Pharm. Bull.

36(11)4554-4559(1988)]

\title{
Photoacoustic Spectroscopic Study of Cyclodextrin-Methyl Orange Systems
}

\author{
Yoshinobu NakaI, * Keiji Yamamoto, Katsuhide Terada, ${ }^{1}$ \\ and MANABU SAKaI
}

Faculty of Pharmaceutical Sciences, Chiba University, 1-33 Yayoicho, Chiba 260, Japan

(Received April 11, 1988)

\begin{abstract}
Photoacoustic (PA) spectral measurements in the visible region were made for methyl orange contained in physical mixtures with $\alpha$ - and $\beta$-cyclodextrins, ground mixtures, and the inclusion compounds. Inclusion of methyl orange in $\beta$-cyclodextrin (molar ratio $1: 1$ ) was confirmed by the Bs type phase solubility diagram. The inclusion compounds of $\alpha$ - and $\beta$-cyclodextrins showed very similar PA patterns $\left(\lambda_{\max }\right.$ at $\left.445 \mathrm{~nm}\right)$ which were different from the pattern of the physical mixture $\left(\lambda_{\max }\right.$ at $\left.395 \mathrm{~nm}\right)$. The physical mixture showed a gradual change of its PA pattern to that of the inclusion type as a function of grinding time. The pattern of the ground mixture was broader than that of the inclusion compound, but became coincident with it after storage at $40 \mathrm{C}$ and $96 \%$ relative humidity for $4 \mathrm{~d}$.

It was concluded that PA spectral measurement is an effective method to investigate inclusion phenomena in the solid state.
\end{abstract}

Keywords_- photoacoustic spectroscopy; methyl orange; cyclodextrin; inclusion; grinding; powder X-ray diffraction

Photoacoustic spectroscopy (PAS) is a new and effective technique to study solid samples because the spectra of solid powdery samples can be measured easily over a wide wavelength region. $^{2-4)}$ In this experiment, the inclusion phenomena of cýclodextrins with methyl orange were studied by PAS.

Cyclodextrins have been used for the improvement of dissolution, bioavailability and stability of drugs in the pharmaceutical field.$^{5.6)}$ The inclusion compounds were prepared by various method, such as co-precipitation, kneading and co-grinding. The co-grinding method is advantageous for preparing inclusion compounds because this method needs no solvents. $^{\left.7{ }^{78}\right)}$ In this experiment, methyl orange was chosen as the guest molecule, since methyl orange is known to form an inclusion compound with $\alpha$-cyclodextrin (molar ratio, methyl orange : $\alpha$-cyclodextrin $=1: 2$ ) in the solid state. ${ }^{9)}$ Ground mixtures of $\alpha$-and $\beta$-cyclodextrins with methyl orange were prepared by using a vibrational mill and the photoacoustic (PA) spectra were measured in order to investigate the molecular state of methyl orange. Furthermore, powder X-ray diffraction patterns of the cyclodextrin-methyl orange systems were measured.

\section{Experimental}

Materials $-\alpha$-Cyclodextrin (Nakarai Chemicals Ltd.) and $\beta$-cyclodextrin (Ando Kasei Co.) were used. Methyl orange and calcium fluoride were purchased from Wako Pure Chemical Ind., Ltd. All reagents were used without further purification.

Visible Absorption Spectroscopy_- Hitachi type 557 and Shimadzu type 200-S spectrometer were used.

Phase Solubility Diagram of the $\boldsymbol{\beta}$-Cyclodextrin-Methyl Orange System - The phase solubility diagram was obtained according to the method of Higuchi and Connors. ${ }^{10)}$ An excess amount of methyl orange was added to $10 \mathrm{ml}$ 
of buffer solution $(0.05 \mathrm{~m}$ sodium carbonate $-0.01 \mathrm{~m}$ sodium bicarbonate buffer, $\mathrm{pH} 8.9)$ containing various amounts of $\beta$-cyclodextrin. The suspensions were shaken at $30 \mathrm{C}$ for $24 \mathrm{~h}$. After attaining dissolution equilibrium, the solution was filtered and the concentration of methyl orange was determined from the absorbance (452 $\mathrm{nm})$ of the solution.

Preparation of Physical Mixture of Cyclodextrin with Methyl Orange - Methyl orange was mixed with dried cyclodextrin in a bottle by shaking. Molar ratios (cyclodextrin: methyl orange) of the prepared mixture were $3: 1$, $2: 1,1: 1$ and $1: 2$.

Preparation of Ground Mixture of Cyclodextrins with Methyl Orange - Dried cyclodextrin and methyl orange were ground together in a vibrational mill (Heiko Seisakusho, TI-200) as reported previously. ${ }^{11)}$ The molar ratios (cyclodextrin: methyl orange) of the mixture were $3: 1,2: 1,1: 1$ and $1: 2$. The total specimen weight was about $3.0 \mathrm{~g}$.

Crystallization of the Ground Mixture-Ground mixture (ground for $10 \mathrm{~min}$ ) was stored at $40{ }^{\circ} \mathrm{C}$ and $96 \%$ relative humidity $(\mathrm{RH})$. Water vapor was generated from saturated potassium sulfate aqueous solution in a desiccator.

Preparation of Inclusion Compounds by the Co-precipitation Method_Cyclodextrin and methyl orange (molar ratio, $\alpha$-cyclodextrin : methyl orange $=2: 1 ; \beta$-cyclodextrin $:$ methyl orange $=1: 1)$ were dissolved in hot water. After cooling, the precipitated crystals were collected by filtration.

Powder X-Ray Diffraction--Measurement of powder X-ray diffraction was done as reported previously. ${ }^{12)}$ The diffractometer was a Rigaku Denki type 2027. The target was $\mathrm{Cu}$, and the detector was a scintillation counter.

Photoacoustic Spectroscopy_-The photoacoustic spectrometer used in this experiment was the same as reported previously. ${ }^{13.14)}$ Relative PA signal of the sample was calculated according to the method of Monahan and Nolle. ${ }^{15 /}$ Samples containing methyl orange were diluted suitably with calcium fluoride in a mortar with a pestle and the PA spectra of the samples were measured.

\section{Results and Discussion}

\section{Phase Solubility Diagram}

The phase solubility diagram of the $\beta$-cyclodextrin-methyl orange system at $30^{\circ} \mathrm{C}$ is shown in Fig. 1. The color change interval of methyl orange was between $\mathrm{pH} 3.1$ and 4.4. The amino group of methyl orange was not charged in alkaline solution (we used $\mathrm{pH} 8.9$ buffer solution). The diagram was of $\mathrm{B}_{\mathrm{S}}$ type, and the insoluble complex was co-precipitated at higher concentration of cyclodextrin. The stoichiometric ratio of the solid complex was calculated as $1: 1$ ( $\beta$-cyclodextrin : methyl orange) form the data in the plateau region. The stoichiometric ratio of the co-precipitated complex was also determined spectrophotometrically to be the same $(1: 1)$ as in the crystallized complex. This result agrees with the data reported by Suzuki and Sasaki. ${ }^{16)}$ Harata has reported that $\alpha$-cyclodextrin also formed an inclusion compound with methyl orange $(\alpha$-cyclodextrin : methyl orange $=2: 1) .{ }^{9)}$ The difference in the stoichiometric ratio between $\alpha$-and $\beta$-cyclodextrins could be ascribed to the size of the cavities.

\section{Comparison of Powder X-Ray Diffractograms}

Figure 2 shows the powder X-ray diffraction patterns of $\alpha$-cyclodextrin-methyl orange systems (molar ratio, $\alpha$-cyclodextrin : methyl orange $=2: 1$ ) and Fig. 3 shows those of $\beta$ cyclodextrin-methyl orange systems (molar ratio, $\beta$-cyclodextrin : methyl orange $=1: 1$ ). The

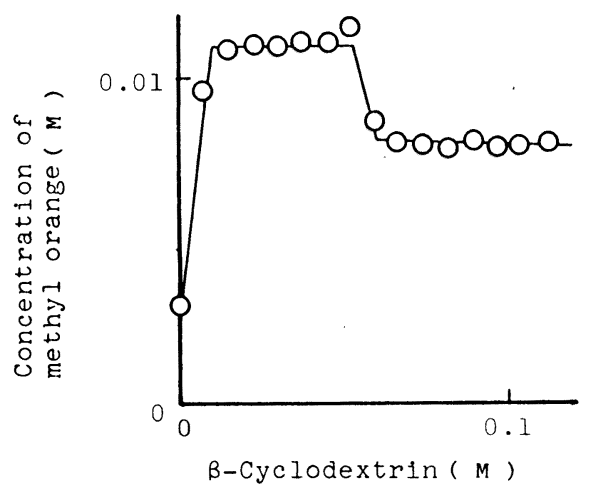

Fig. 1. Phase Solubility Diagram of $\beta$-Cyclodextrin-Methyl Orange System in Buffer Solution $(\mathrm{pH} 8.9)$ at $30^{\circ} \mathrm{C}$ 

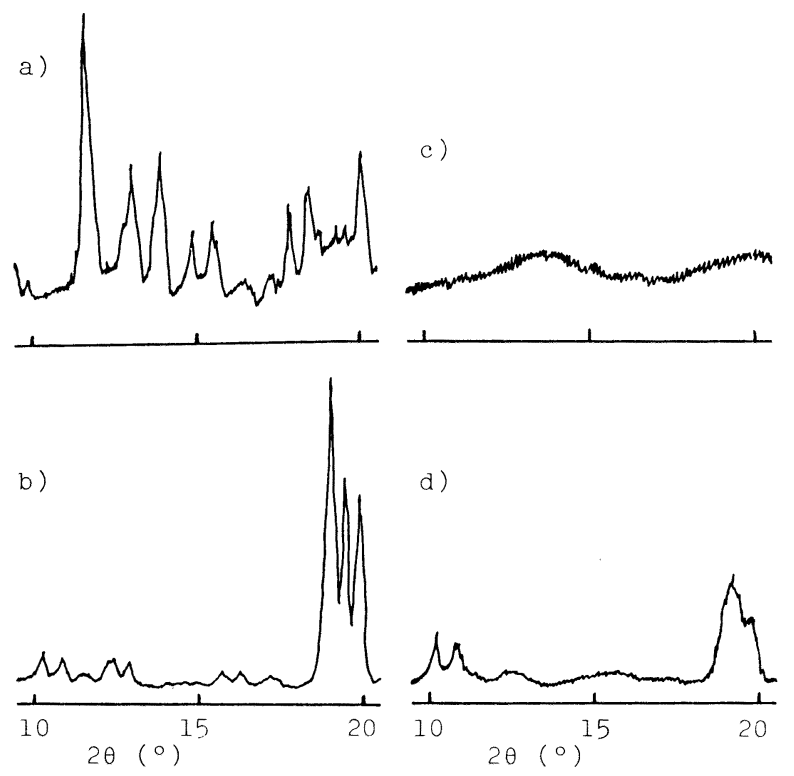

Fig. 2. Powder X-Ray Diffraction Patterns of $\alpha$-Cyclodextrin-Methyl Orange Systems (Molar Ratio, $\alpha$-Cyclodextrin : Methyl Orange $=$ $2: 1)$

a), physical mixture of $x$-cyclodextrin with methyl orange; b), co-precipitated compound of $\alpha$-cyclodextrin-methyl orange; c), ground mixture of $x$-cyclodextrin with methyl orange; d), crystallized mixture of $\alpha$-cyclodextrin with methyl orange after grinding (stored at $400^{\circ} \mathrm{C}, 96^{\circ} \mathrm{RH}$ ).
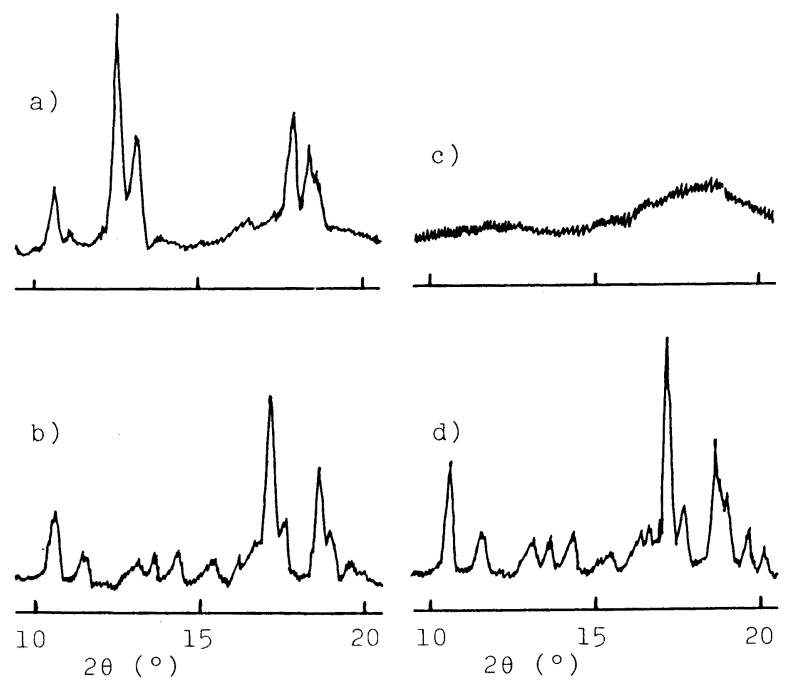

Fig. 3. Powder X-Ray Diffraction Patterns of $\beta$-Cyclodextrin-Methyl Orange Systems (Molar Ratio, $\beta$-Cyclodextrin: Methyl Orange= $1: 1)$

a), physical mixture of $\beta$-cyclodextrin with methyl orange; $b$ ), co-precipitated compound of $\beta$-cyclodextrin-methyl orange; $c$ ), ground mixture of $\beta$-cyclodextrin with methyl orange; d), crystallized mixture of $\beta$-cyclodextrin with methyl orange after grinding (stored at $40{ }^{\circ} \mathrm{C}, 96 \% \mathrm{RH}$ ).

X-ray diffraction patterns of the physical mixtures showed sharp crystalline peaks (Figs 2a, 3a) which were due to the crystals of cyclodextrin and methyl orange. The co-precipitated samples of $\alpha$ - and $\beta$-cyclodextrin showed different diffraction patterns from the physical mixtures (Figs 2b, 3b). It was confirmed that the co-precipitated samples were the inclusion compounds by comparing the diffraction patterns with those of the crystalline inclusion compounds. ${ }^{9)}$ The ground mixture (ground for $10 \mathrm{~min}$ ) showed halo patterns (Figs. 2c, 3c) as reported previously, ${ }^{11)}$ indicating that the samples were in the amorphous state. When the ground mixture were stored at $40{ }^{\circ} \mathrm{C}$ and $96 \% \mathrm{RH}$ for $4 \mathrm{~d}$, the X-ray diffraction peaks due to the inclusion compound appeared in the patterns as shown in Figs. $2 \mathrm{~d}$ and $3 \mathrm{~d}$. This suggested that the ground mixture crystallized in the form of the inclusion compound during storage at high $\mathrm{RH}^{8)}$

\section{Photoacoustic Spectroscopic Studies}

The PA spectrum of methyl orange with calcium fluoride, an inert diluent having 


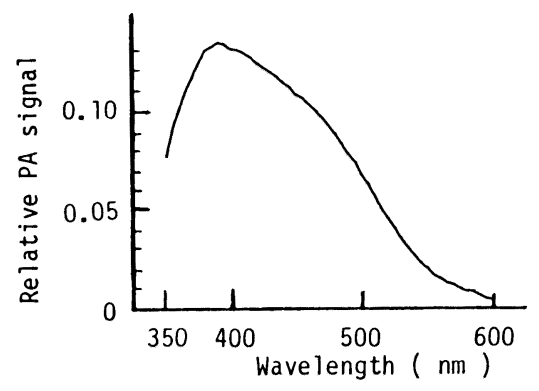

Fig. 4. Photoacoustic Spectrum of Methyl Orange Diluted with Calcium Fluoride
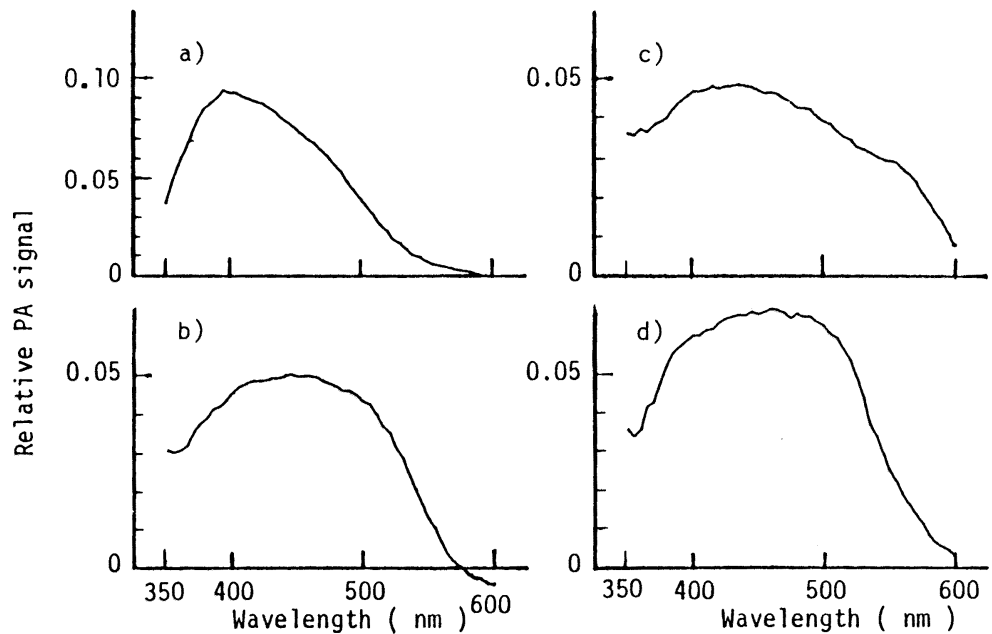

Fig. 5. Photoacoustic Spectra of $\alpha$-Cyclodextrin-Methyl Orange Systems (Molar Ratio, $\alpha$-Cyclodextrin : Methyl Orange $=2: 1$ )

a), physical mixture of $\alpha$-cyclodextrin with methyl orange; b), co-precipitated compound of $\alpha$-cyclodextrin-methyl orange; c), ground mixture of $\alpha$-cyclodextrin with methyl orange; d), crystallized mixture of $\alpha$-cyclodextrin with methyl orange after grinding (stored at $40^{\circ} \mathrm{C}$, $96 \% \mathrm{RH})$

negligible absorption, is shown in Fig. 4. The PA peak was observed at $390-395 \mathrm{~nm}$.

Photoacoustic spectra of the $\alpha$-cyclodextrin-methyl orange systems are shown in Fig. 5 and those of the $\beta$-cyclodextrin-methyl orange systems are shown in Fig. 6.

The physical mixture (Figs. 5a, 6a) showed the same pattern as methyl orange crystals (Fig. 4) which had a PA peak at $395 \mathrm{~nm}$, while the inclusion compounds had a peak at $445 \mathrm{~nm}$ (Figs. 5b, 6b). This difference in the peak position between the physical mixtures and the inclusion compounds may be attributed to the difference of the molecular state of methyl orange.

Figures $5 \mathrm{c}$ and $6 \mathrm{c}$ show the PA spectra of the ground mixtures (ground for $10 \mathrm{~min}$ ) of cyclodextrin-methyl orange systems. These spectral patterns were entirely different from those of the physical mixtures, and rather resembled the patterns of the inclusion compounds. It is considered therefore that methyl orange molecules were included in cyclodextrin molecules during grinding. An incompletely included structure was estimated for the ground mixture on the basis that the ground mixtures showed broader spectral patterns than the inclusion compounds. After storage of the ground mixtures at $40{ }^{\circ} \mathrm{C}, 96 \% \mathrm{RH}$ for $4 \mathrm{~d}$, the PA spectra coincided with those of the inclusion compounds. This suggested that the inclusion compounds crystallized at $40{ }^{\circ} \mathrm{C}, 96 \% \mathrm{RH}$ from the ground mixtures, in agreement with the powder X-ray diffraction results. 

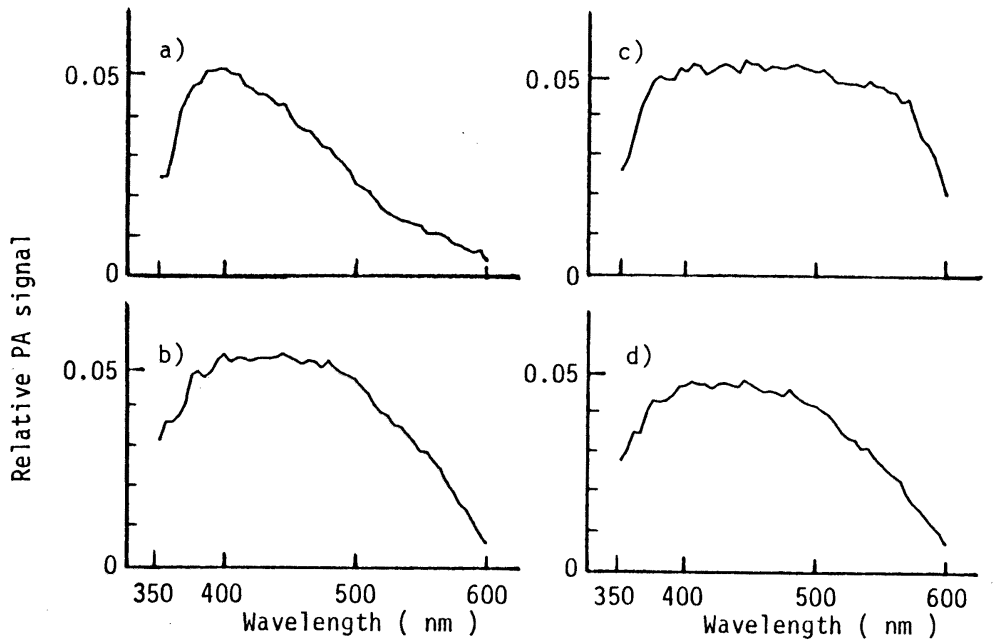

Fig. 6. Photoacoustic Spectra of $\beta$-Cyclodextrin-Methyl Orange Systems (Molar Ratio, $\beta$-Cyclodextrin: Methyl Orange $=1: 1$ )

a), physical mixture of $\beta$-cyclodextrin with methyl orange; b), co-precipitated compound of $\beta$-cyclodextrin-methyl orange; $\mathrm{c}$, ground mixture of $\beta$-cyclodextrin with methyl orange; d), crystallized mixture of $\beta$-cyclodextrin with methyl orange after grinding (stored at $40 \mathrm{C}$, $96 \%$ RH).
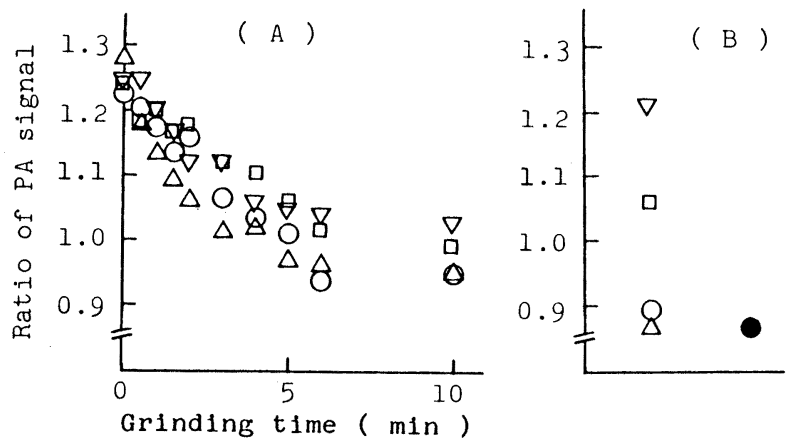

Fig. 7. Change of the Ratio of PA Signals $\left(\mathbf{P A}_{395} / \mathbf{P A}_{445}\right)$ of Ground Mixtures as a Function of Grinding Time (A), and the Ratio of the Inclusion Compound and the Crystallized Mixtures after $10 \mathrm{~min}$ of Grinding (B)

Molar ratio of $\alpha$-cyclodextrin : methyl orange: $\triangle$. $3: 1 ; \bigcirc, 2: 1 ; \square, 1: 1 ; \nabla, 1: 2 ; 0$, inclusion compound prepared by co-precipitation method.

In order to investigate the PA spectral change during grinding, the ratio of the relative PA signal at $445 \mathrm{~nm}\left(\lambda_{\max }\right.$ of the inclusion compound) to the relative PA signal at $395 \mathrm{~nm}\left(\lambda_{\max }\right.$ of the physical mixture) was plotted versus the grinding time (Fig. 7). In the case of the $\alpha$ cyclodextrin-methyl orange systems (molar ratio, $\alpha$-cyclodextrin : methyl orange $=2: 1$ ), the initial ratio of the relative PA signals $\left(\mathrm{PA}_{395} / \mathrm{PA}_{445}\right)$ was 1.22 . The ratio decreased with grinding time and leveled off at 0.944 after $10 \mathrm{~min}$ of grinding. When the ratio of cyclodextrin to the drug was increased, the decrease in the ratio of PA signals became clearer.

The ratios of relative PA signal of the crystallized samples were different from those of the ground mixtures. In the cases of the molar ratio of $\alpha$-cyclodextrin : methyl orange $=2: 1$ and $3: 1$ systems, the ratio of relative PA signal of the crystallized samples was the same as that of the inclusion compound. In the molar ratio of $\alpha$-cyclodextrin : methyl orange $=1: 1$ and $1: 2$ systems, however, the PA signal ratio of crystallized samples were higher than that of the inclusion compound. This result suggests that the crystallized samples of molar ratio of $1: 1$ and $1: 2$ (larger than the inclusion ratio of $2: 1$ ) contain methyl orange molecules that are not included in the cyclodextrin.

In the case of $\beta$-cyclodextrin-methyl orange systems, the $\mathrm{PA}$ signal ratio $\left(\mathrm{PA}_{395} / \mathrm{PA}_{445}\right)$ 

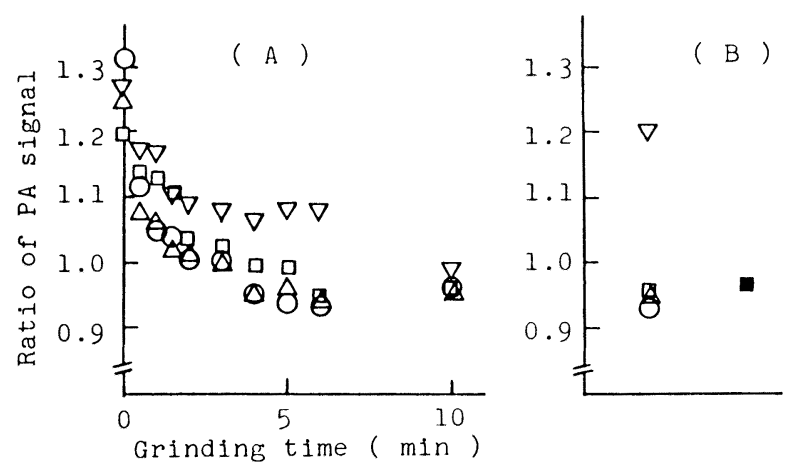

Fig. 8. Change of the Ratio of PA Signals $\left(\mathrm{PA}_{395} / \mathrm{PA}_{445}\right)$ of Ground Mixtures as a Function of Grinding Time (A), and the Ratio of the Inclusion Compound and the Crystallized Mixtures after $10 \mathrm{~min}$ of Grinding (B)

Molar ratio of $\beta$-cyclodextrin : methyl orange: $\triangle$, $3: 1 ; \bigcirc, 2: 1 ; \square, 1: 1 ; \nabla, 1: 2 ; \boldsymbol{\square}$, inclusion compound prepared by co-precipitation method.

decreased with increasing grinding time in the same way as in the $\alpha$-cyclodextrin-methyl orange systems (Fig. 8). As the inclusion compound consisted of a $1: 1$ stoichiometric ratio of $\beta$-cyclodextrin to methyl orange, all the PA signal ratio of the crystallized samples having various molar ratio were almost the same as that of the inclusion compound except for the $1: 2$ mixture. In the case of $\beta$-cyclodextrin : methyl orange $=1: 2$, excess methyl orange could separate from the monomolecular dispersed state because the PA signal ratio became large.

In conclusion, it was possible to determine whether methyl orange molecules were in the complexed form or in the free form from the PA spectra. Therefore, PAS seems to be a convenient method to investigate the inclusion phenomena of cyclodextrin in the solid state.

\section{References and Notes}

1) Present address: Faculty of Pharmaceutical Sciences, Toho University, 2-2-1, Miyama, Funabashi, Chiba 274, Japan.

2) Y. Pao (ed.), "Optoacoustic Spectroscopy and Detection," Academic Press, Inc., New York, 1977.

3) A. Rosencwaig, "Photoacoustics and Photoacoustic Spectroscopy," John Wiley and Sons, Inc., New York, 1980.

4) P. Ganguly and C. N. R. Rao, Proc. Indian Acad. Sci. (Chem. Sci.), 90, 153 (1981).

5) M. L. Bender and M. Komiyama, "Cyclodextrin Chemistry," Springer Verlag, Berlin, 1978.

6) J. Szejtli, "Cyclodextrins and Their Inclusion Complexes," Akademiai Kiado, Budapest, 1982.

7) Y. Nakai, K. Yamamoto, K. Terada, and K. Akimoto, Chem. Pharm. Bull., 32, 685 (1984).

8) K. Kawano and Y. Nakai, Yakugaku Zasshi, 103, 1060 (1983).

9) K. Harata, Bull. Chem. Soc. Jpn., 49. 1493 (1976).

10) T. Higuchi and K. A. Connors, Anal. Chem. Inst., 4, 117 (1965).

11) Y. Nakai, S. Nakajima, K. Yamamoto, K. Terada, and T. Konno, Chem. Pharm. Bull., 26, 3419 (1978).

12) Y. Nakai, K. Yamamoto, K. Terada, and Y. Ueno, Chem. Pharm. Bull., 34, 315 (1986).

13) Y. Nakai, K. Yamamoto, K. Terada, and M. Sakai, Chem. Pharm. Bull., 33, 3068 (1985).

14) Y. Nakai, K. Yamamoto, K. Terada, and M. Sakai, Chem. Pharm. Bull., 35, 4255 (1987).

15) E. M. Monahan, Jr. and A. M. Nolle, J. Appl. Phys., 48, 3519 (1977).

16) M. Suzuki and Y. Sasaki, Chem. Pharm. Bull., 27, 609 (1979). 